\title{
Can mean $A D C$ value and $A D C$ ratio of benign prostate tissue to prostate cancer assist in the prediction of clinically significant prostate cancer within the PI- RADSv2 scoring system?
}

\author{
Samar Ramzy Ragheb* and Reem Hassan Bassiouny
}

\begin{abstract}
Background: The aim of this study is to investigate whether quantitative DW metrics can provide additive value to the reliable categorization of lesions within existing PI-RADSv2 guidelines. Fifty-eight patients with clinically suspicious prostate cancer who underwent PR examination, PSA serum levels, sextant TRUS-guided biopsies, and biparametric MR imaging were included in the study.

Results: Sixty-six lesions were detected by histopathological analysis of surgical specimens. The mean ADC values were significantly lower in tumor than non-tumor tissue. The mean ADC value inversely correlated with Gleason score of tumors with a significant $p$ value $<0.001$.Conversely, a positive relationship was found between the ADC ratio (ADC of benign prostatic tissue to prostate cancer) and the pathologic Gleason score with a significant elevation of the ADC ratio along with an increase of the pathologic Gleason score $(p<0.001)$. ROC curves constructed for the tumor $A D C$ and $A D C$ ratio helped to distinguish pathologically aggressive (Gleason score $\geq 7$ ) from non-aggressive (Gleason score $\leq 6$ ) tumors and to correlate it with PIRADSv2 scoring to predict the presence of clinically significant PCA (PIRADSv2 DW $\geq 4$ ). The ability of the tumor ADC and ADC ratio to predict highly aggressive tumors (GS> 7) was high (AUC for ADC and ADC ratio, 0.946 and 0.897; $p=0.014$ and 0.039 , respectively). The ADC cut-off value for $G S \geq 7$ was $<0.7725$ and for $G S \leq 6$ was $>0.8620$ with sensitivity and specificity 97 and 94\%. The cutoff ADC ratio for predicting (GS > 7) was 1.42 and for GS $\leq 6$ was $>1.320$ with sensitivity and specificity 97 and 92\%. By applying this ADC ratio cut-off value the sensitivity and specificity of reader 1 for correct categorization of PIRADSv2 DW > 4 increased from 90 and $68 \%$ to 95 and $90 \%$ and that of reader 2 increased from 94 and $88 \%$ to 97 and 92\%, respectively.
\end{abstract}

Conclusion: Estimation of DW metrics (ADC and ADC ratio between benign prostatic tissue and prostate cancer) allow the non-invasive assessment of biological aggressiveness of prostate cancer and allow reliable application of the PIRADSv2 scoring to determine clinically significant cancer (DW score > 4) which may contribute in planning initial treatment strategies.

Keywords: Diffusion weight MRI, Gleason score, ADC, PIRADSv2, Prostatic cancer

\footnotetext{
* Correspondence: doc mina2004@yahoo.com

Department of Radiodiagnosis, Faculty of Medicine, Ain Shams University, Cairo, Egypt
}

\section{Springer Open}

(c) The Author(s). 2020 Open Access This article is licensed under a Creative Commons Attribution 4.0 International License, which permits use, sharing, adaptation, distribution and reproduction in any medium or format, as long as you give appropriate credit to the original author(s) and the source, provide a link to the Creative Commons licence, and indicate if changes were made. The images or other third party material in this article are included in the article's Creative Commons licence, unless indicated otherwise in a credit line to the material. If material is not included in the article's Creative Commons licence and your intended use is not permitted by statutory regulation or exceeds the permitted use, you will need to obtain permission directly from the copyright holder. To view a copy of this licence, visit http://creativecommons.org/licenses/by/4.0/. 


\section{Background}

Prostate cancer $(\mathrm{PCa})$ is the most common solid malignant neoplasm among men in the USA and Western Europe with a considerably high mortality and morbidity [1]. The diagnosis of prostate cancer traditionally relies on digital rectal examination and estimation of serum prostate-specific antigen (PSA) followed by transrectal ultrasound (TRUS)-guided biopsy [2, 3]. Today with the use of multi-parametric magnetic resonance imaging (mp-MRI), accurate diagnosis and grading of prostatic cancer has become increasingly popular. It includes a combination of $\mathrm{T} 2$-weighted, diffusion weighted, and dynamic contrast-enhanced imaging $[4,5]$.

The Prostate Imaging Reporting and Data System (PIRADS) classification for prostate MRI was first introduced in 2012 and has been revised and simplified in 2015 and is still evolving [6]. The specific aims of PIRADS 2 were to establish guidelines for minimum acceptable technical parameters in multi-parametric prostate MRI, to simplify and standardize the terminology and content of mp-MRI reports, and to develop assessment categories that summarize the levels of suspicion or risk of having significant prostatic cancer [7]. In the Prostate Imaging Reporting and Data System (PI-RADS) version 2, a zone-based dominant sequence classification system is used to assign MRI visible lesions a final category from 1 to 5 that represents the like hood of clinically significant lesions [8]. Diffusion weighted imaging (DWI) plays a pivotal role in PIRADSv2, serving as the primary sequence for evaluation of the peripheral zone (PZ) and the secondary sequence for the transition zone (TZ) [9]. A PI-RADSv2 assessment category of DW > 4 implies a clinically significant prostate cancer (CSC), which is pathologically defined as a tumor with a Gleason score of 7 or more. A score of $\geq 4$ on DWI becomes a final PI-RADSv2 score of $\geq 4$ in the PZ, regardless of the findings on dynamic contrast-enhanced (DCE) imaging [9]. Even for the TZ, DWI enables a final determination of clinically significant cancer (CSC) when the findings of T2-weighted imaging (T2WI) are inconclusive (e.g., a score of 3 on T2WI) [9].The role of dynamic contrast enhancement in routine evaluation of prostate cancer is uncertain. At the present time its value in addition to $\mathrm{T} 2 \mathrm{~W}$ and diffusion sequence is considered modest. In the PIRADS version 2 suspicious enhancements (focal early enhancement) is considered a minor feature in evaluation [10].

The PI-RADSv2 still has several limitations including its inability to detect small clinically significant cancer (CSC) and the limited inter-reader reproducibility [11, 12]. The inter-reader variability is attributed to the fact that all scoring criteria in this system are subjective and rely on qualitative evaluation $[12,13]$. Accordingly quantitative parameters derived from DWI like the apparent diffusion coefficient (ADC), the ADC ratio (ratio between benign prostatic tissue and prostate cancer), and the normalized ADC value (ratio of tumor to non-tumor tissue) have been tested to improve the reproducibility of PI-RADSv2via reducing inter-reader variability [14-17]. However, quantitative DW metrics have not been widely incorporated into the PI-RADSv2 guidelines, largely over concern that different MRI units may produce different ADC values [18].

In this study, we tried to investigate whether the use of the mean $\mathrm{ADC}$ values and $\mathrm{ADC}$ ratio can improve interpretation of the lesions and reduce inter-reader disagreement within the PIRADSv2 scoring system of prostate cancer.

\section{Methods}

Between January 2017 and July 2019, a total of 58 consecutive patients with newly diagnosed histologically proven prostate cancer (median 64 years; range 45-84) were enrolled in the study. This prospective study was approved by the institutional review board of our institution and written informed consent was obtained from all patients to use their data for research purpose. Inclusion criteria were (1) all patients with elevated PSA values greater than $4 \mathrm{ng} / \mathrm{mL}$ who underwent sextant TRUSguided biopsies. (2) All patients who underwent preoperative bi-parametric MRI of the prostate, (3) all patients surgically treated by radical prostatectomy, (4) pathologic localization of lesions using a 24-sector map. The patients' age and PSA levels are listed (in Table 1). Exclusion criteria were (1) patients who received antiandrogen treatment, (2) patients having contraindication to MRI, and (3) patients with poor image quality and (4) lesions located outside the peripheral zone.

Table 1 Patient characteristics

\begin{tabular}{ll}
\hline Parameter & Value \\
\hline Age (years), median (range) & 64 years (45-84) \\
PSA (ng/mL) median (range) & $44.8(10.90-1725.0 \mathrm{ng} / \mathrm{mL})$ \\
Tumor location & 66 \\
PZ & $60(90 \%)$ \\
TZ and CZ & $6(10 \%)$ \\
Gleason score & \\
6 & $11(19 \%)$ \\
7 & 25 \\
$8-9-10$ & 30 \\
ECE & 24 \\
SVI & 9 \\
Tumor volume (cm3), median (range) & $1.8(0.1-26.0)$
\end{tabular}

$P S A$ prostate-specific antigen, $P Z$ peripheral zone, $T Z$ transition zone, $G S$ Gleason score, $E C E$ extracapsular extension, SVI seminal vesicle invasion 


\section{MR imaging}

MR imaging was performed using a Philips achieva XR 1.5-T system, Baltimore, Netherland, Holland, and a torso XL 16 channels phased array body coil. The examination was done before or at least 3 weeks after transrectal ultrasound-guided biopsy to minimize post biopsy artifacts, and radical prostatectomy was conducted within a median time of 2 months of the MR examination (range $0.5-4)$. In order to reduce bowel peristalsis, an intramuscular injection of $20 \mathrm{mg}$ of butyl scopolamine was given to the patient before starting the scan. A bi-parametric MR imaging protocol was performed and consisted of multi-planar turbo spinecho T1-weighted images, turbo spin-echo T2-weighted images 3D fast field-echo and echo-planar DWI. Dynamic contrast enhanced MRI was not performed in order to reduce the cost and duration of the study particularly considering its modest role in evaluation of newly diagnosed prostate cancer and its minor contribution in lesion characterization within the PIRDAS version 2 system

The T1-weighted images were acquired in the axial plane primarily to exclude the presence of post biopsy hemorrhage that may mimic or conceal cancer.The T2weighted turbo spin-echo images in the axial, sagittal, and coronal planes were acquired with the following imaging parameters: TR range/TE range, 2690-3800/8090; slice thickness, $3 \mathrm{~mm}$; inter-slice gap, $0.3-1 \mathrm{~mm}$; 512 $\times 304$ matrix; field of view (FOV), $180 \times 119$; number of signals acquired (NSA), 6 ; sensitivity-encoding (SENSE) factor, 2; slice number, 36; and acquisition time of each plane, $6 \mathrm{~min}$. DW images were acquired using the single-shot echo-planar imaging technique. The image plane was axial perpendicular to the long axis of the prostate from base to apex. The scanning parameters were as follows: TR 2740-3000; TE 83-85; slice thickness, $3 \mathrm{~mm}$; inter-slice gap, $1 \mathrm{~mm}$; matrix, $112 \times 110$; FOV, $20 \mathrm{~cm}$; SENSE factor, 2; and NSA, 6. 1.9-3.0 mm in frequency-encoding direction and $1.9 \mathrm{~mm}$ in phaseencoding direction;Diffusion-encoding gradients were applied using three $b$ values of 0,300 , and $1000 \mathrm{~s} / \mathrm{mm}^{2}$. The acquisition time of DWI was within $3 \mathrm{~min}$. ADC maps were automatically constructed on a pixel by-pixel basis.

\section{Pathologic examination consensus}

All surgical specimens were examined by the same two genitourinary pathologists and any disagreement was resolved by consensus. Whole-mount specimens of prostate gland were processed utilizing section thickness of 4-8 mm. Mapping included visual reporting of (1) tumor location, (2) tumor volume, (3) extracapsular extension, and (4) seminal vesicle invasion. The index lesion was defined as the cancer focus showing the largest volume or the one showing extraprostatic extension.
The 24-sector map was utilized to record the location of the lesions. According to histopathologic documentation, lesions were classified as clinically significant (Gleason score $\geq 7$, including $3+4$ with prominent but not predominant Gleason 4 component; tumor volume $\geq 0.5$ $\mathrm{cm}^{3}$; extraprostatic extension or a combination of these features versus an insignificant PCa based on the modified Epstein criteria; (Gleason score $(\mathrm{GS})<7$, tumor volume $<0.5 \mathrm{~cm}^{3}$, and organ-confined PCa [19].

\section{MRI data analysis}

Two genitourinary radiologists with 7- and 10-year experience in interpretation of prostate MRI respectively analyzed the MR images independently. Both were blinded to the histopathologic results. Prostate cancer has been identified on MR images on the basis of the following criteria; T2W hypointense nodular foci in the peripheral zone or a very pronounced T2W hypointense sickle-shaped lesion in the transitional zone of the prostate glandthat showed high DWI signal intensity than the rest of the prostate on the highest $b$ value images corresponding to low signal intensity on ADC map images. In the transition zone, the PI-RADS assessment category of a lesion is determined primarily on T2Wimages and correlated to DWI/ADC. An equivocal lesion in the transitional zone (PI-RADS category 3 ) is assigned to PI-RADS category 4 if the DWI corresponds with category 5 (markedly intense greater than $1.5 \mathrm{~cm}$ ). The lesion remains assigned to PI-RADS category 3 if the DWI corresponds to DWI category 4 (markedly intense but less than $1.5 \mathrm{~cm}$ ) or a lower category.

The ADC map was sometimes used to identify the lesions not well identified by T2 and DWI. Lesion location was recorded using the pathologic tumor sector map.The DWIs were analyzed for PI-RADSv2 scoring using image viewing software (Sante DICOM Viewer Pro).

The website of the American College of Radiology for PIRADSv2 (http://www.acr.org/Quality-Safety/Resources/PIR ADS) was used as the reference for DWI scoring of PIRADSv2 $[9,20]$. ROIs were drawn manually on the visualized lesion to include as much of the inner aspect of the lesion as possible without contracting the edges. While drawing the ROIs, the neurovascular bundle and urethra were excluded to minimize any error in the calculation of the ADC.A second ROI of similar size was drawn on the same MRI slice in a region contra-lateral to the identified lesion to include benign tissue within the same prostate zone in the same relative location. The ADC value of each lesion was calculated within the same image-viewing software. The $\mathrm{ADC}$ ratio of benign prostatic tissue to $\mathrm{PCa}$ was estimated for each lesion by the two radiologists independently. The DWI scores of PI-RADSv2 of cancerous lesions were then reported and revised. 


\section{Statistical analysis}

Data were coded and entered using the statistical package SPSS (Statistical Package for the Social Sciences) version 23. The weighted kappa was evaluated to assess inter-reader agreement in PI-RADSv2 DWI scoring between the two readers.Quantitative data were analyzed using mean, standard deviation, minimum, and maximum values for categorical data. The relationship between the tumor ADC value and ADC ratio with the surgical biopsy Gleason score was analyzed using linear regression model.

ROC curves were constructed with area under curve analysis performed to detect best cut-off value of ADC and ADC ratio for differentiating pathologically aggressive(Gleason score $\geq 7$ ) from non-aggressive (Gleason score $\leq 6)$ tumors and to correlate it with PIRADSv2. $p$ values less than 0.05 were considered statistically significant.

\section{Results}

In the 58 patients enrolled in the study (Table 1), 66 PCa lesions were detected by histopathologic analysis of the surgical specimens. Ninety-one percent $(60 / 66)$ of the lesions were located in the peripheral zone, and 9\% $(6 / 66)$ were located in the transitional zone. The histopathological type of the diagnosed prostate cancer was adenocarcinoma in all lesions. Eighty-nine percent (59/ 66) of the lesions were clearly identified on the T2 WI. Localization of the lesions was then confirmed on the DWI sequences.

Histopathological analysis reported 11(16.6\%) lesions to have Gleason score $(3+3), 11(16.6 \%)$ to have Gleason score $(3+4), 14(21 \%)$ have Gleason score $(4+3)$, $12(18 \%)$ had Gleason score $(4+4), 10(15.2 \%)$ had Gleason score $(5+4), 5(7.5 \%)$ had Gleason score $(4+5)$, and $3(4.5 \%)$ had Gleason score $(5+5)$. In the $5(7.6 \%)$ lesions with negative bi-parametric-MRI findings (false negative), the histologic analysis showed a biopsy Gleason score of $3+3$, indicating a well-differentiated tumor, with two or fewer pathologic cores, each one containing $10 \%$ or less malignant cells.

\section{Assessment of inter-reader agreement for PI-RADSv2 DWI score}

Among the 66 pathologically detected lesions (Table 2), 54 lesions were clinically significant. Using the PIRA DSv2 DW reader 1 detected 91\% (50/54) of clinically significant lesions with score overall score of 4 or 5 , and 16 lesions with score 2 or 3 . Reader 2 detected $94.5 \%$ $(52 / 54)$ of clinically significant lesions and 14 lesions with score 2 or 3 (Table 2). Inter-reader agreement was satisfactory for PI-RADSv2 DWI score 4 or 5 (weighted kappa $=0.678 ; 95 \%$ CI, 0.490-0.882). Reader 1 falsely assigned two benign lesions as prostate carcinoma: one was focal prostatitis, and the second was a nodule of stromal benign prostatic hyperplasia $(\mathrm{BPH})$ and reader 2 falsely reported a small abscess as PCA. There was disagreement for overall PI-RADSv2 DWI score in 6/ $55(11 \%)$ of clinically significant lesions (Figs. 1 and 2).

\section{Quantitative lesion characteristics}

The mean ADC values were significantly lower in tumor (Figs. 3, 4, 5, 6, and 7) than non-tumor tissue in the various regions of the gland; in the PZ (0.842 versus $1.138)$ and in TZ (0.795 versus 1.212$)$ with a significant $p$ value in both regions (Table 3 ). The mean ADC value of the tumors has a strong positive relationship of multiple $R=0.96$ with their Gleason score (Table 4). The mean ADC value of the tumor has a positive relationship of multiple $R=0.86$ with Gleason score of different grades of the tumor in the peripheral zone (Table 5).We also found that the mean ADC of Gleason score $(4+3)$ and Gleason score $(3+4)$ has a very strong positive relationship of multiple $R$ and $R^{2}=1$ (Table 6). Also, a positive relationship (multiple $R=$ 0.87) was found between the ADC ratio and the pathologic Gleason score with a significant elevation of the $\mathrm{ADC}$ ratio along with an increase of the pathologic Gleason score (Table 5).

ROC curves drawn for the tumor $\mathrm{ADC}$ and $\mathrm{ADC}$ ratio were reliable in distinguishing pathologically aggressive (Gleason score $\geq 7$ ) from non-aggressive (Gleason score $\leq 6)$ tumors and to correlate it with PIRADSv2 scoring to predict the presence of clinically significant PCA (PIRADSv2 DW $\geq 4$ ). The ability of the tumor ADC and ADC ratio to predict highly aggressive tumors $(\mathrm{GS}>7$ ) was high (AUC for ADC and ADC ratio, 0.946 and $0.897 ; p=0.014$ and 0.039 , respectively). The ADC cutoff value for GS $\geq 7$ was $<0.7725$ and for GS $\leq 6$ was > 0.8620 with sensitivity and specificity $97 \%$ and $94 \%$. The cut-off ADC ratio for predicting (GS > 7) was 1.42 with sensitivity and specificity $97 \%$ and $92 \%$.By applying this ADC ratio cut-off value the sensitivity and specificity of reader 1 for correct categorization of PIRADSv2 DW > 4 increased from $90 \%$ and $68 \%$ to $95 \%$ and $90 \%$ and that of reader 2 increased from $94 \%$ and $88 \%$ to 97 and $92 \%$ respectively. Inter-reader agreement for quantitative ADC measurement for PI-RADSv2 DWI score 4 or 5 was satisfactory (weighted kappa $=0.718$ )

T2-weighted imaging increases sensitivity and specificity for detecting PCA. This may be attributed to increased contrast on DWI images compared with conventional imaging [21]. When combined with T2weighted imaging, the sensitivity and specificity for PC range from 71 to $89 \%$ and 61 to $91 \%$ respectively, compared with $51-86 \%$ and $60-84 \%$, respectively, for T2WI alone $[22,23]$. In our study about $12 \%$ of the lesions were not well identified by $\mathrm{T} 2$ (these lesions were 
Table 2 PIRADS2 scoring system using DWI for PZ lesions and T2-weighted for TZ lesions

\begin{tabular}{|c|c|c|c|}
\hline & & $\begin{array}{l}\text { Reader } 1 \text { No of } \\
\text { cases }\end{array}$ & $\begin{array}{l}\text { Reader } 2 \text { No of } \\
\text { cases }\end{array}$ \\
\hline \multicolumn{4}{|c|}{ PIRADS-score (DW finding in PZ an TZ lesions) } \\
\hline 1 & No abnormality (i.e., normal) on ADC and high $b$ value DWI & 0 & 0 \\
\hline 2 & Indistinct hypointense area on ADC map & 7 & 8 \\
\hline 3 & $\begin{array}{l}\text { Focal moderate diffusion restriction with focal mildly/moderately hypointense on ADC and isointense/ } \\
\text { mildly hyperintense on high } b \text { value DWI }\end{array}$ & 9 & 6 \\
\hline 4 & $\begin{array}{l}\text { Focal marked diffusion restriction with markedly hypointense on } A D C \text { and markedly hyperintense on high } \\
b \text { value } \mathrm{DWl} ;<1.5 \mathrm{~cm} \text { in greatest dimension }\end{array}$ & 26 & 25 \\
\hline 5 & Same as 4 but $\geq 1.5 \mathrm{~cm}$ in greatest dimension or definite extraprostatic extension/invasive behavior & 20 & 23 \\
\hline \multicolumn{4}{|c|}{ PIRADS-score (T2W finding for TZ lesions) } \\
\hline 1 & Homogeneous intermediate signal intensity (normal & 0 & 0 \\
\hline 2 & Circumscribed hypointense or heterogeneous encapsulated nodule(s) (BPH) & 1 & 1 \\
\hline 3 & Heterogeneous signal intensity with obscured margins Includes others that do not qualify as 2, 4, or 5 & 1 & 2 \\
\hline 4 & $\begin{array}{l}\text { Lenticular or non-circumscribed, homogeneous, moderately hypointense, and }<1.5 \mathrm{~cm} \text { in greatest } \\
\text { dimension }\end{array}$ & 3 & 2 \\
\hline 5 & Same as 4 , but $\geq 1.5 \mathrm{~cm}$ in greatest dimension or definite extraprostatic extension/invasive behavior & 1 & 1 \\
\hline
\end{tabular}

predominantly located in the $\mathrm{CZ}$ and $\mathrm{TZ}$ ) and many of these lesions were better delineated by DW. However, still $7.5 \%$ of lesions could not be identified on the DW images. We agreed with the previous results obtained by Tan et al. [24] regarding the limitation of $\mathrm{T} 2$ in $\mathrm{TZ}$ tumors and limitation of DWI in both TZ tumors and prostatic base tumors. Evaluation of tumors in prostatic base by DWI can be limited by increased cellularity in the normal prostatic base.
The current PIRADSv2 DW recommendations for prostate cancer reporting rely mainly on the subjective evaluation of lesion DW signal intensity. The qualitative scoring of PIRADSv2 is based on the recognition of a difference in restricted diffusion in the prostate gland with utilization of terms such as moderately hypointense and markedly hypointense [9, 10, 12]. Accordingly, the current PIRADSv2 suffers a high degree of inter-reader discrepancy. Agreement among highly experienced

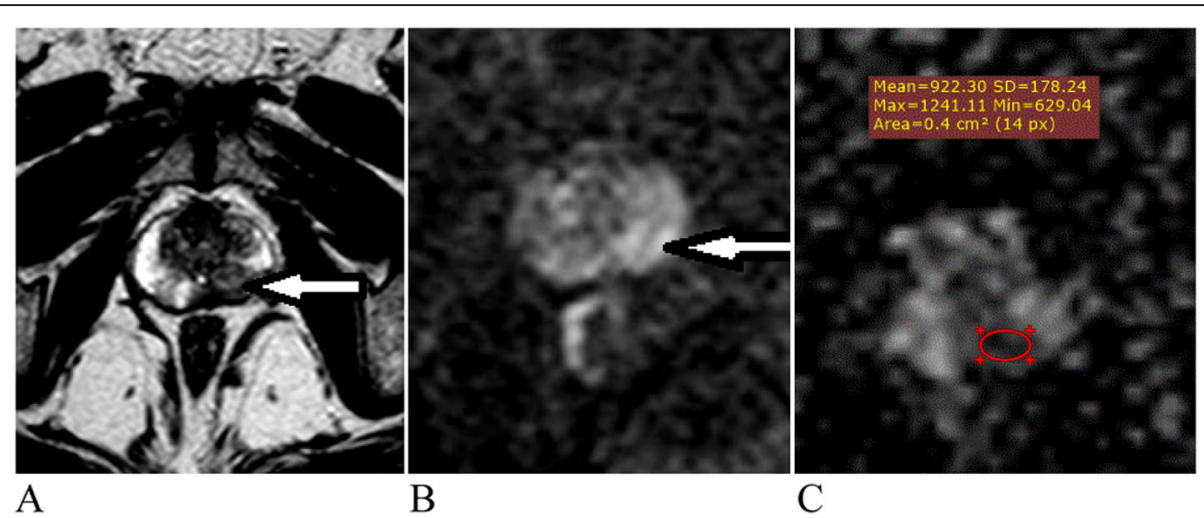

Fig. 1 A 67-year-old man with PSA 16 with discordant interpretation in terms of PIRADSv2 DWI score. a Axial T2 showing a moderately hypointense lesion located dorsally in the left peripheral zone of the apex not abutting the capsule. $\mathbf{b}$ DWI of $b 800$ shows a focal area of moderate hyperintensity, measuring $1.1 \mathrm{~cm}$, in left PZ (arrow). CADC map also shows low signal intensity of the lesion with mean ADC value 0.922. The ADC ratio was 1.33. The qualitative DWI score was 4 for reader 1 and 3 for reader 2 . Histopathology was Adenocarcinoma GS $3+3=$ 6; tumor volume $=1.1 \mathrm{~cm}^{3}$; organ-confined) 


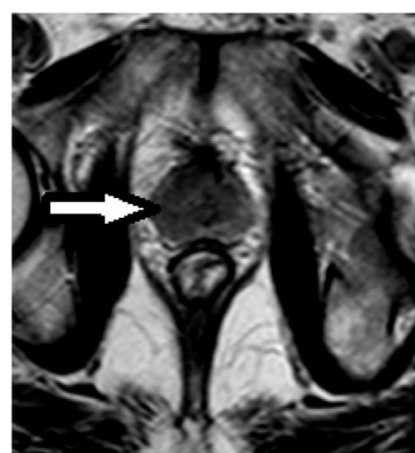

A

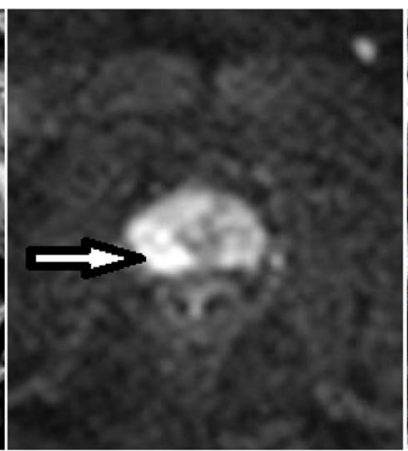

B

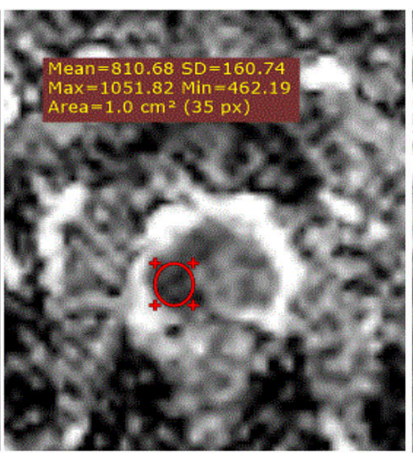

C

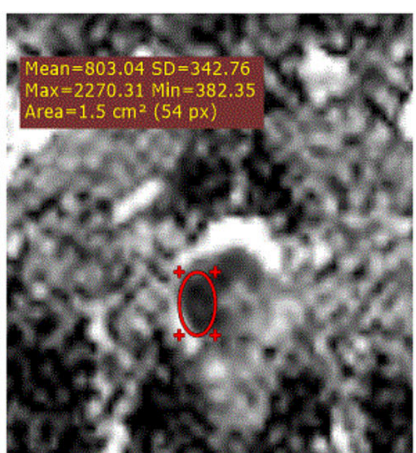

D

Fig. 2 A 70-year-old man with total PSA 35 with discordant interpretation in terms of PIRADSv2 DWI score. a Axial T2 showing bilateral diffuse (non-circumscribed) low signal intensity of the peripheral zone near the apex. b DWI (800) showing bilateral focal bright signal intensity lesions in PZ (not markedly hyperintense). c, $\mathbf{d}$ ADC map also shows low signal intensity of the lesions with mean ADC value 0.782 . The ADC ratio between benign prostatic tissues was 1.63. The qualitative DWI score was 4 for reader 1 and 3 for reader 2 histopathology was adenocarcinoma GS $4+3$ $=7)$
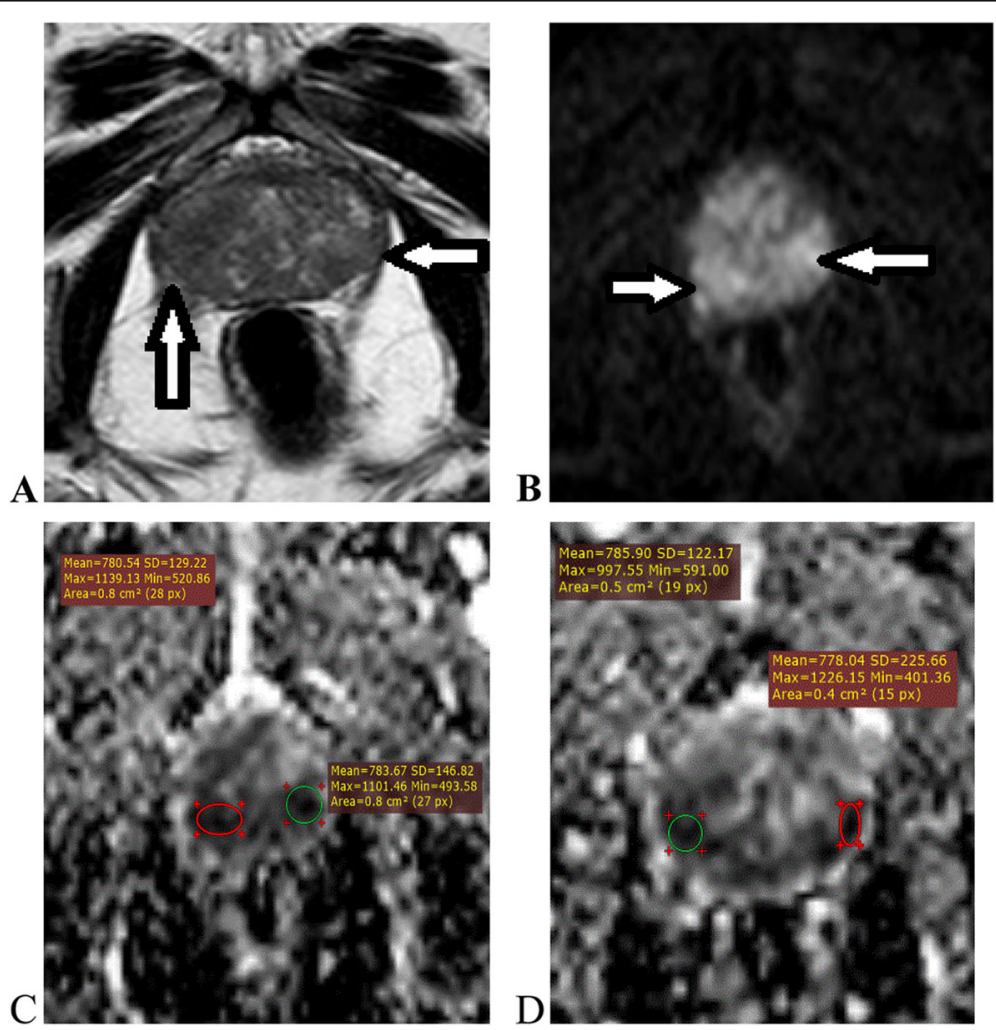

Fig. 3 A 64-year-old man with total PSA 68 with concordant interpretation in terms of PIRADSv2 DWI score. a Axial T2 of the apex showing right peripheral zone non circumscribed homogenous low signal intensity lesion $<1.5 \mathrm{~cm}$. b DWI of $b 800$ shows a markedly hyperintense focal lesion in right PZ (arrow). CADC map also shows marked hypointensity of the lesion with mean ADC value 0.803. The qualitative DWI score was 4 for readers 1 and 2 . The ADC ratio between benign prostatic tissues was 1.58 . Histopathology was adenocarcinoma GS $3+4=7 ;$ tumor volume $=$ $1.5 \mathrm{~cm}^{3}$; organ-confined) 

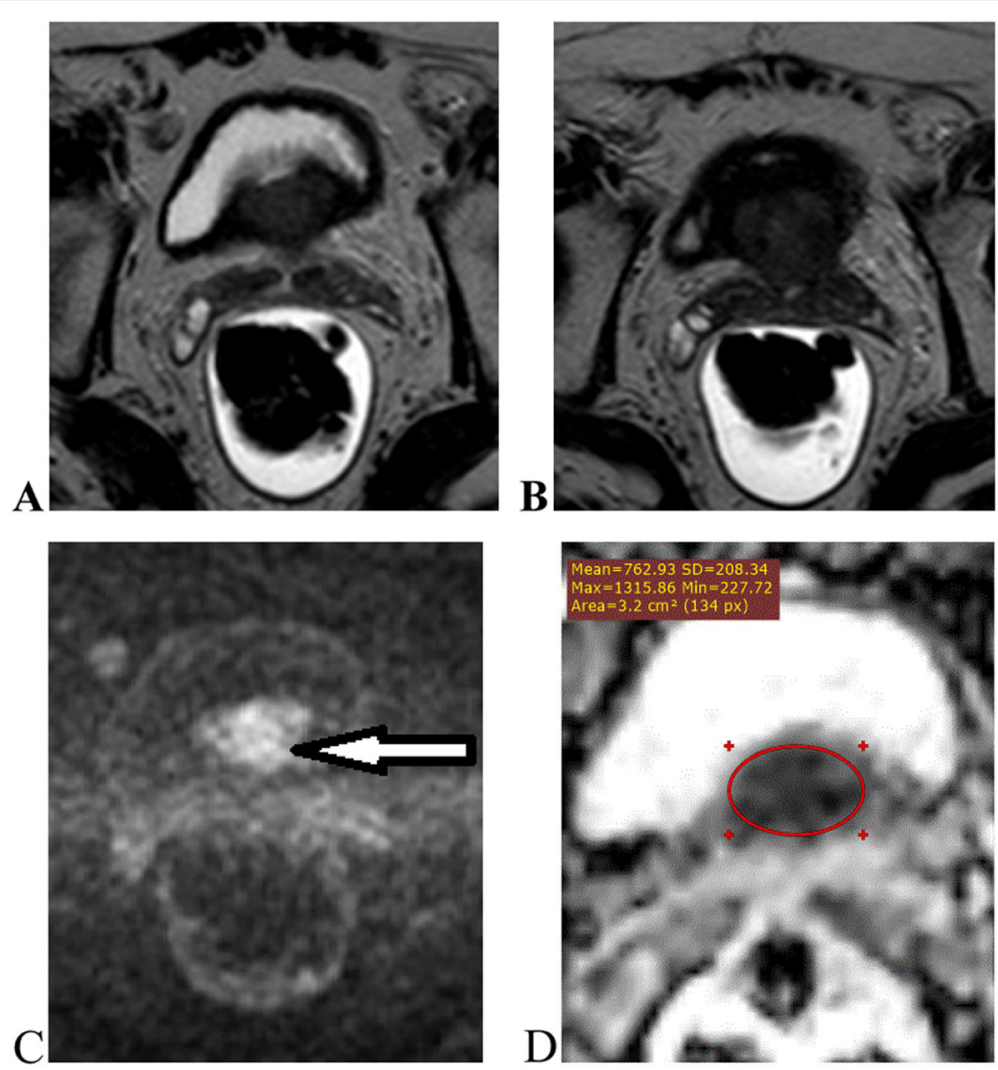

Fig. 4 A 78-year-old man with total PSA 83 with concordant interpretation in terms of PIRADSV2 DWI score. a, b Axial T2W with a central zone isointense signal intensity lesion that was not well identified on T2W images but protruding into bladder. c DWI (at $b$ value 800 ) showing marked bright signal intensity of the lesion $1.5 \mathrm{~cm}$. $\mathbf{d}$ ADC map image showing marked low signal of the lesion with mean ADC value 0.762 . The qualitative DWI score was 4 for readers 1 and 2. The ADC ratio between benign prostatic tissues was 1.67. Histopathology was Adenocarcinoma GS $4+4=7$; tumor volume $=1.2 \mathrm{~cm}^{3}$; organ-confined)

readers has been reported to be as low as $51 \%$ and between moderately experienced readers as $53 \%[25,26]$. Inter-reader agreement for PI-RADS may be affected by the variability among readers in manipulating the display conditions [27], the difference in radiologists' level of experience [28] or familiarity with local imaging protocols [29]. In addition, DWI may underestimate CSC with small tumor volume (i.e., less than $0.5-1.0 \mathrm{~cm}^{3}$ ) [30, 31]. Furthermore, there may be significant variability in ADC values across vendors or MR protocols [32, 33]. In our study, inter-reader agreement was satisfactory in patients for PI-RADSv2 DWI score $\geq 4$ with disagreement in only $11 \%(6 / 55)$ of cases. This could be due to stabilization of the display conditions in the image viewing software, relatively large tumors volume and high percentage of CSC as well as relatively close level of experience of the two readers.

In concordance with previous studies, current study results confirmed that the mean ADC value of prostate cancer lesions is significantly lower than that of the normal prostatic tissue [34-36]. However, because tissue diffusivity depends on both biological (patient's age and body temperature) and technical factors ( $b$ value, location, and size of the region of interest), no definite ADC cut-off for tumors has been established to date [34].

Current study results confirmed the inverse relationship between the ADC value and the GS [35-37]. Doo et al. [38] evaluated the substantial value of combining DWI with T2-weighted imaging to detect low- (GS, $\leq 6)$ from intermediate- or high-risk (GS, $\geq 7$ ) prostate cancer. They found that the addition of DWI to T2weighted imaging improves the accuracy of detecting intermediate- or high-grade prostate cancers, but not for low-grade prostate cancer detection. Similarly Somford et al. [39] and Cornud et al. [40] showed that the relationship between ADC and GS enabled the prediction of high-grade tumor. Oto et al. [17] instead found a substantial overlap between the ADC values of tumors with different GS; however, they concluded that ADC values of tumors with a GS of 6 and those of tumors with a GS of 9 were relatively well separated. The results of current study went more with those of Hambrock et al. [14] and 


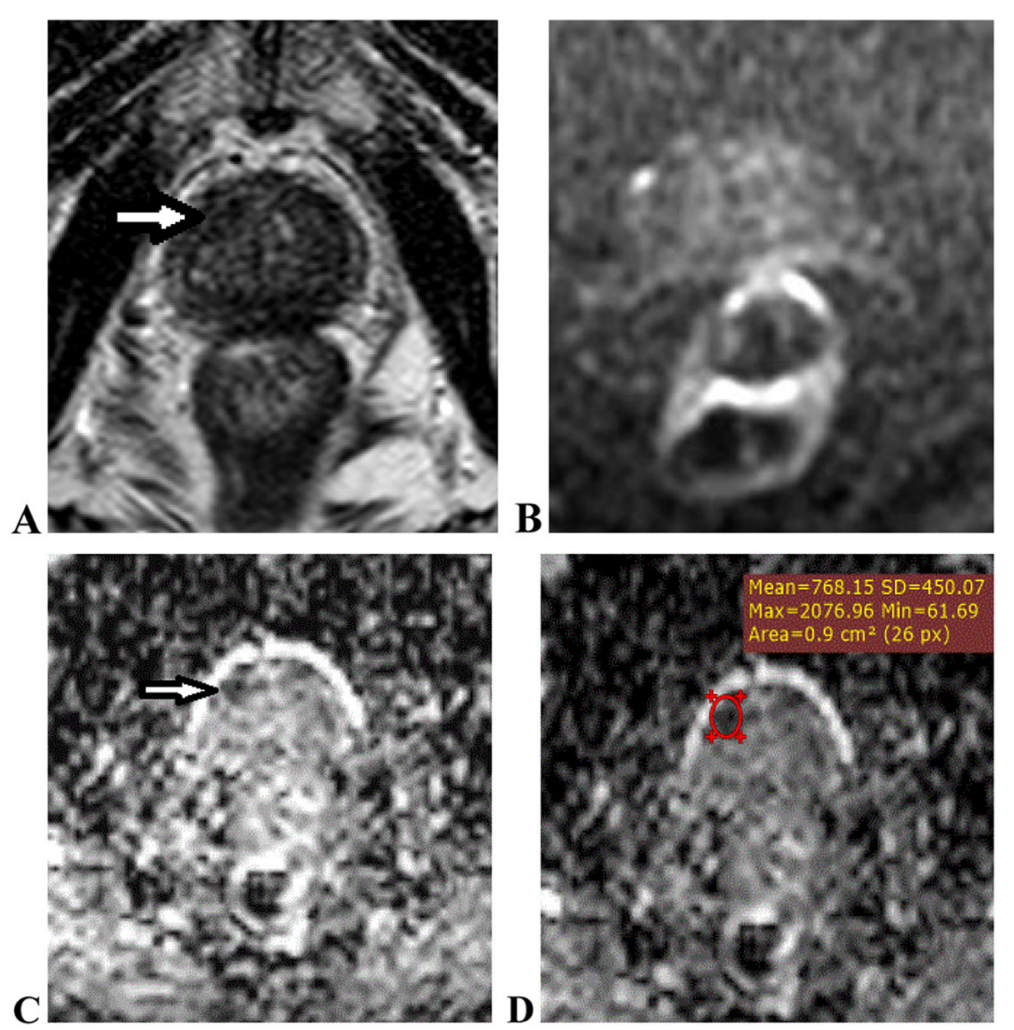

Fig. 5 A 76-year-old male patient with total PSA 318with discordant interpretation in terms of PIRADSV2 DWI score. a Axial T2 showing small hardly defined right sided transitional zone slightly low signal intensity lesion. B-DWI (at b value 800) the lesion was not well identified. b, $\mathbf{d}$ ADC map images showing mild low signal intensity of the lesion with mean ADC value 0.768. The DWI score was 3 for reader 1 and 3 for reader 2 . The ADC ratio between benign prostatic tissues was 1.65. Histopathology was Adenocarcinoma GS $4+4=6$; tumor volume $=0.6$ $\mathrm{cm}^{3}$; organ-confined)

Woodfield et al. [41] in that DWI could achieve a high discriminatory performance in the differentiation between low-, intermediate-, and high-grade cancer. The mean ADC value of low grade (Gleason score, $\leq 6)$ was $0.919 \mathrm{~mm}^{2} / \mathrm{s}$, of intermediate grade (Gleason score 7) was 0.799 and of high grade (Gleason score, $\geq 8$ ) tumors was 0.716 ( $p$ value $<0.001)$.

In a larger study by Nowak et al. [42], they calculated ADC cut-off values for different criteria for maximum values of both sensitivity (90.5\%) and specificity (62.5\%), ADC values lower than $1.005 \times 10 \_3 \mathrm{~mm}^{2} / \mathrm{s}$ indicated a GS $>7$, for high sensitivity $(95.2 \%)$ and specificity of $50 \%$, the cut-off ADC value for GS > 7 was $1.052 \times 10 \_3$ $\mathrm{mm}^{2} / \mathrm{s}$ and ADC of $>0.762 \times 10 \_3 \mathrm{~mm}^{2} / \mathrm{s}$ indicated rather a $3+4$ type Gleason grade with an AUC of $69.6 \%$, corresponding to a sensitivity and specificity of $77.5 \%$ and $64.7 \%$ respectively.

In a study by Park et al. [16], they found that the best cutoff ADC ratio for determining a DWI score $\geq 4$ was greater than 1.3 for both readers. With the use of this $\mathrm{ADC}$ ratio, the rate of inter reader disagreement declined by about half (from 11.8 to $5.9-6.0 \%$ in PCa; from
13.8 to $7.0-7.1 \%$ in PZ cancer) and the PPV for the presence of CSC was $100 \%$.

In current study, the ROC curve analysis showed that the ability of the tumor ADC and ADC ratio to predict highly aggressive tumors (GS > 7) was high (AUC for $\mathrm{ADC}$ and $\mathrm{ADC}$ ratio, 0.946 and $0.897 ; p=0.014$ and 0.039 , respectively). The ADC cut-off value for GS $\geq 7$ was $<0.7725$ and for GS $\leq 6$ was $>0.8620$ with sensitivity and specificity $97 \%$ and $94 \%$. The cut-off ADC ratio for predicting (GS > 7) was 1.42 and for GS $\leq 6$ was > 1.320 with sensitivity and specificity $97 \%$ and $92 \%$. By applying this ADC ratio cut-off value, the sensitivity and specificity of reader 1 for correct categorization of PIRA DSv2 DW > 4 increased from $90 \%$ and $68 \%$ to $95 \%$ and $90 \%$ and that of reader 2 increased from $94 \%$ and $88 \%$ to 97 and $92 \%$, respectively.

Accordingly, the addition of ADC ratio can improve the reliable prediction of clinically significant cancer (DW score $\geq 4$ ) within the PIARDASv2 system. The ADC ratio can be widely applied for several reasons. First, the ADC ratio is simple to measure using the ROI. Second, the ADC ratio is not affected by display 

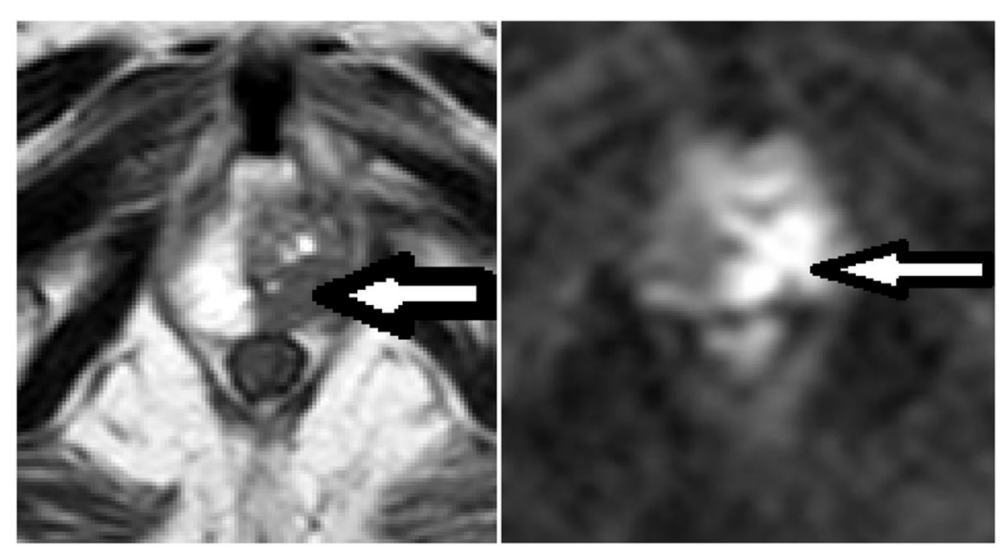

A

B

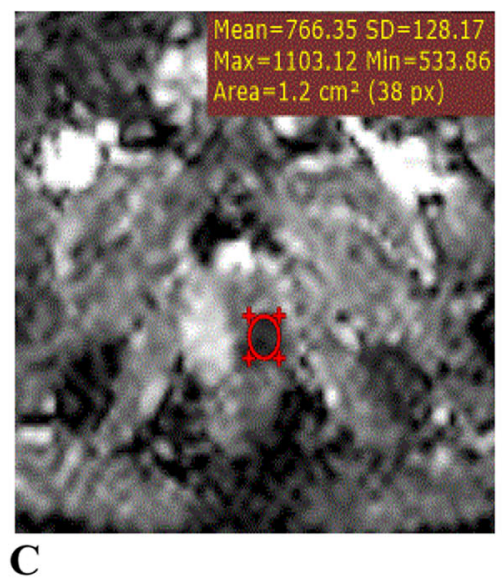

Fig. 6 A 73-year-old male patient with total PSA 169 with concordant interpretation in terms of PIRADSv2 DWI score. a Axial T2 showing left peripheral zone low signal intensity lesion $1.7 \mathrm{~cm}$ with wide base abutting the capsule. b DWI (at $b$ value 800) showing marked bright signal intensity of the lesion. c ADC map image showing marked low signal intensity of the lesion with mean ADC value 0.766 . The DWI score was 5 for readers 1 and 2. The ADC ratio between benign prostatic tissues was 1.66. Histopathology was adenocarcinoma GS $4+4=8$; tumor volume $=$ $1.6 \mathrm{~cm}^{3}$; organ-confined)

conditions (e.g., window width or level). Third, the ADC ratio can be standardized across different MR protocols or vendors, because the ADCs of tumor and non-tumor tissue will change proportionally. Therefore, a universal ADC ratio cut-off may be applicable in the future for discriminating significant from non-significant PCa [16].

Our study has multiple limitations. First, because of the limited sample size, this study has to be considered a preliminary study warranting further verification in a larger patient population. Second, lack of discrimination between the measurements taken in the various anatomic locations of the prostate whether in the peripheral or transitional zones makes this quantitative analysis deficient compared to previous studies which analyzed the DW parameters in each zone separately; however, this was due to the very limited number of lesions within the $\mathrm{TZ}$ and CZ. Third, low and intermediate $b$ values were used in our study but according to the previous literatures there is no consensus on the optimal $b$ value for
DWI of the prostate. Although studies have been made with high DWI values of ( $b 2000 \mathrm{~s} \backslash \mathrm{mm}$ ) recent studies showed that the ADC map constructed with high values of (b $2000 \mathrm{~s} \backslash \mathrm{mm}$ ) did not add significant information to differentiate prostate cancer from benign tissue compared to lower values of $b[43,44]$. Fourth, the number of low-grade $\mathrm{PCa}$ or clinically insignificant $\mathrm{PCa}$ was so small which could have interfered with the accuracy of quantitative measurement for discrimination between low and high grade tumors.

\section{Conclusion}

In conclusion, the quantitative analysis of diffusion weighted metrics (ADC and ADC ratio between benign prostatic tissues and prostate cancer provide significant additive value to the PIRADSv2 scoring system. Applying threshold values for ADC and ADC ratio yielded excellent interpretation of DW score $\geq 4$ and decreased the level of inter-reader disagreement. 


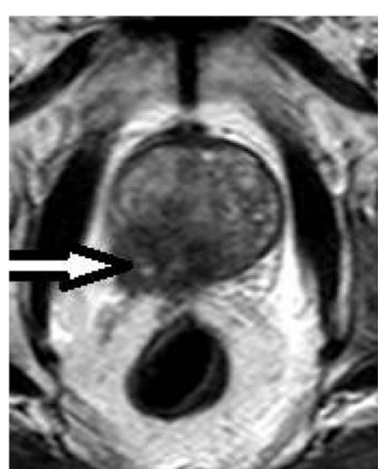

A

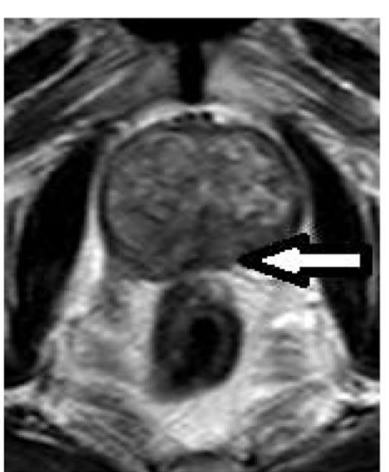

B

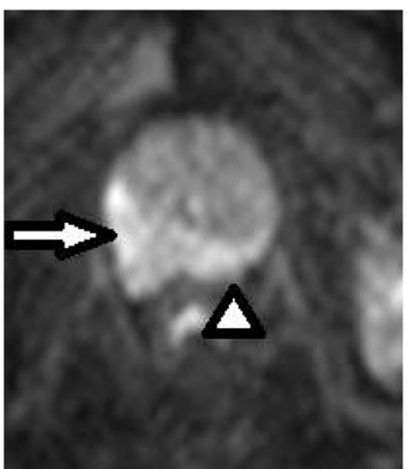

C
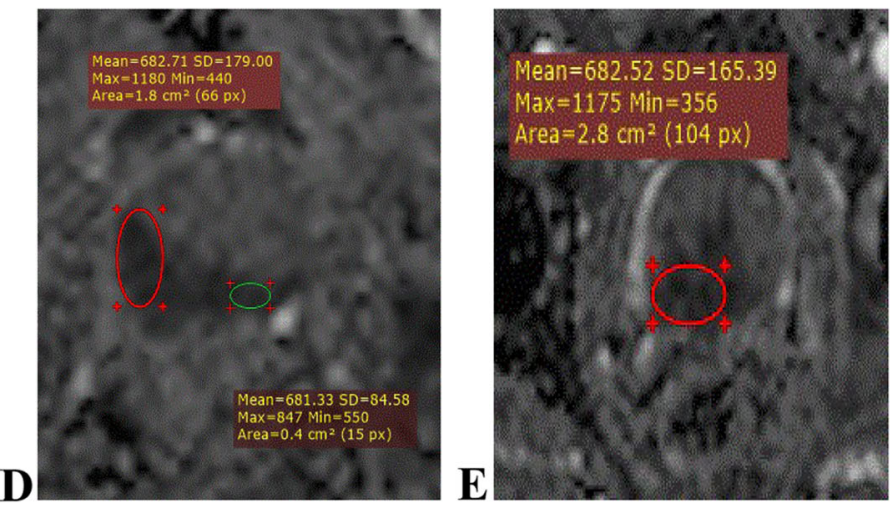

Fig. 7 A 75-year-old male patient with total PSA 91 with concordant interpretation in terms of PIRADSv2 DWI score. a, b Axial T2 showing bilateral peripheral zone low signal intensity lesions with extra prostatic extension with right neurovascular bundle involvement. c DWI (at $b$ value 600) showing bright signal of the lesions. $\mathbf{d}$, e ADC map images showing marked low signal intensity of the lesions with mean ADC value 0.682. The DWI score was 5 for readers 1 and 2. The ADC ratio between benign prostatic tissues was 1.868. Histopathology was adenocarcinoma GS $5+$ $4=9$; tumor volume $=1.9 \mathrm{~cm}^{3}$; organ-confined)

Table 3 Mean ADC value

\begin{tabular}{lll}
\hline & Mean ADC value & \\
\cline { 2 - 3 } & Peripheral zone & Transition zone \\
\hline Tumor & $0.842 \pm 0.007$ & $0.795 \pm 0.001$ \\
Non-tumor tissue & $1.138 \pm 0.003$ & $1.212 \pm 0.002$ \\
$\boldsymbol{p}$ value & $<0.0001$ & $<0.0001$ \\
\hline
\end{tabular}

Table 4 Demonstrating the mean ADC value and the standard deviation of each Gleason score in the peripheral zone

\begin{tabular}{lllllllll}
\hline \multicolumn{8}{c}{ Gleason score } \\
\cline { 2 - 8 } & & $\mathbf{3 + 3}$ & $\mathbf{3}+4$ & $\mathbf{4}+3$ & $4+4$ & $4+5$ & $5+4$ & $5+5$ \\
\hline Mean & Mean & 0.919 & 0.812 & 0.781 & 0.765 & 0.710 & 0.679 & 0.633 \\
ADC & SD & \pm & \pm & \pm & \pm & \pm & \pm & \\
& & 0.006 & 0.002 & 0.003 & 0.003 & 0.008 & 0.007 & \\
\hline
\end{tabular}

Multiple $R=0.96, R^{2}=0.93$
Table 5 Demonstrating the mean ADC value and the standard deviation of different grades of the tumor in the peripheral zone

\begin{tabular}{lllll}
\hline & & GS & \\
\cline { 3 - 5 } & & 6 & 7 & $8-10$ \\
\hline MEAN ADC & Mean $^{\mathrm{a}}$ & 0.919 & 0.799 & 0.716 \\
& Standard deviation & \pm 0.006 & \pm 0.016 & \pm 0.046 \\
ADC ratio $^{\mathrm{b}}$ & & 1.386 & 1.594 & 1.779 \\
\hline
\end{tabular}

aultiple $R=0.86, R^{2}=0.75$

${ }^{\text {b }}$ Multiple $R=0.87, R^{2}=0.77$

Table 6 Demonstrating the difference between the mean ADC value and the standard deviation of Gleason score $3+4$ and Gleason score $4+3$

\begin{tabular}{llll}
\hline & & \multicolumn{2}{l}{ Gleason score } \\
\cline { 3 - 4 } & & $3+4$ & $4+3$ \\
\hline ADC & Mean & 0.812 & 0.781 \\
& Standard deviation & & \\
\hline
\end{tabular}




\section{Abbreviations}

CSC: Clinically significant cancer; CZ: Central zone; DCE: Dynamic contrastenhanced; DWI: Diffusion weighted imaging; ESUR: European Society of Urogenital Radiology; mpMRI: Multi-parametric magnetic resonance imaging; MRI: Magnetic resonance imaging; PI-RADS: Prostate Imaging Reporting and Data System; Pca: Prostate cancer; PZ: Peripheral zone; PSA: Serum prostate specific antigen; T2WI: T2-weighted imaging; TRUS: Transrectal ultrasoundguided biopsy; TZ : Transition zone

\section{Acknowledgements}

Not applicable

\section{Authors' contributions}

$\mathrm{RH}$ conceived and designed the analysis. SR participated in collecting the data and performing the analysis and wrote the manuscript. All authors read and approved the final manuscript.

\section{Funding}

The authors state that this work has not received any funding.

\section{Availability of data and materials}

The datasets used and/or analyzed during the current study available from the corresponding author on reasonable request.

\section{Ethics approval and consent to participate}

Institutional review board (at the Faculty of Medicine, Ain Shams University, Cairo, Egypt) approval was obtained in June 2016, but no number was given at that time. Written informed consent was obtained from all patients.

\section{Consent for publication}

Patients included in this research gave written informed consent to publish the data contained within this study.

\section{Competing interests}

The authors declare that they have no competing interests.

Received: 3 June 2020 Accepted: 1 November 2020

Published online: 30 November 2020

\section{References}

1. Jemal A, Siegel R, Xu J, Ward E et al (2010) Cancer statistics, 2010. CA Cancer J Clin 60:277-300

2. Lilja H, Ulmert D, Vickers AJ (2008) Prostate-specific antigen and prostate cancer: prediction, detection and monitoring. Nat Rev Cancer 8:268-278

3. Puech P et al (2014) Prostate cancer: diagnosis, parametric imaging and standardized report. Diagn Interv Imaging 95:743-752

4. Sala E, Akin O, Moskowitz CS et al (2006) Endorectal MR imaging in the evaluation of seminal vesicle invasion; diagnostic accuracy and multivariate feature analysis. Radiology 238:929-937

5. Siddiqui MM, Rais-Bahrami S, Turbey B et al (2015) Comparison of MR/ ultrasound fusion guided biopsy with ultrasound guided biopsy for the diagnosis of prostatic cancer. JAMA 313:390-397

6. Coakley FV, Oto A, Alexander LF, Allen BC, Davis BJ, Froemming AT et al (2017) ACR appropriateness criteria prostate cancer-pretreatment detection, staging, and surveillance. J Am Coll Radiol 14(5S):S245-S257

7. El-Samei HA, Amin FA, Hassan EE et al (2016) Assessment of the accuracy of multi-parametric MRI with PI-RADS 2.0 scoring system in the discrimination of suspicious prostatic focal lesions. Egypt J Radiol Nucl Med 46:1075-1082

8. Weinreb JC, Barentsz JO, Chyke PL et al (2016) PIRADS prostate imaging reporting and data system 2015, version2. Eur Urol 69:16-40

9. Barentsz JO, Weinreb JC, Verma S et al (2016) Synopsis of the PIRADS V2 guidelines for multiparametric prostate magnetic resonance imaging and recommendations for use. Eur Urol 69:41-49

10. Weinreb JC, Barentsz JO, Choyke PL et al (2016) PI-RADS prostate imaging reporting and data system: 2015, version 2. Eur Urol 69:16-40

11. Ruprecht $\mathrm{O}$, Weisser $\mathrm{P}$, Bodelle B, Ackermann H, Vogl TJ (2012) MRI of the prostate: interobserver agreement compared with histopathologic outcome after radical prostatectomy. Eur J Radiol 81:456-460

12. Rosenkrantz AB, Oto A, Turbey B, Westphalen AC et al (2016) Prostate imaging reporting and date system (PIRADS) version2: critical look. AJR Am J Roentgenol 206:1179-1183
13. Rosenkrantz AB, Rabb JS, Taneja SS, Ream JM et al (2017) Proposed adjustments to PIRADS version2 decision rules: impact on prostate cancer detection. Radiology 283:119-129

14. Hambrock T, Somford DM, Huisman HJ, van Oort IM, Witjes JA, Hulsbergenvan de Kaa CA et al (2011) Relationship between apparent diffusion coefficients at 3.0-T MR imaging and Gleason grade in peripheral zone prostate cancer. Radiology 259(2):453-461

15. Verma S, Rajesh A, Morales H, Lemen L, Bills G, Delworth M et al (2011) Assessment of aggressiveness of prostate cancer: correlation of apparent diffusion coefficient with histologic grade after radical prostatectomy. AJR Am J Roentgenol 196(2):374-381

16. Park SY, Shin SJ, Jung DH, Cho NH, Choi YD, Rha KH, Hong SJ, Taik Oh Y et al (2016) PI-RADS version 2: quantitative analysis aids reliable interpretation of diffusion-weighted imaging for prostate cancer. Eur Radiol 27(7):27762783

17. Oto A, Yang C, Kayhan A et al (2011) Diffusion weighted and dynamic contrast-enhanced MRI of prostate cancer: correlation of quantitativeMR parameters with Gleason score and tumor angiogenesis. Am J Roentgenol 197:1382-1390

18. Ploussard G, Epstein J, Montironi R et al (2011) The contemporary concept of significant versus insignificant prostate cancer. Eur Urol 60:291-303

19. American College of Radiology. PI-RADS v2. (2016): http://www.acr.org/ Quality-Safety/Resources/PIRADS

20. Gaur S, Harmon S, Rosenbulum L, Greer MD et al (2018) Can apparent diffusion coefficient values assist PI-RADS version 2 DWI scoring? A correlation study using the PI-RADSv2 and International Society of Urological Pathology Systems. AJR Am J Roentgenol 211:114-120

21. Choi YJ, Kim JK, Kim N, Kim KW, Choi EK, Cho KS et al (2007) Functional MR imaging of prostate cancer. Radiographics 27:63-77

22. Kajihara H, Hayashida Y, Murakami R, Katahira K, Nishimura R, Hamada Y, Kitani K, Kitaoka M, Suzuki Y, Kitajima M, Hirai T, Morishita S, Awai K, Yamashita $Y$ et al (2009) Usefulness of diffusion-weighted imaging in the localization of prostate cancer. Int J Radiat Oncol Biol Phys 74:399-403

23. Yoshimitsu K, Kiyoshima K, Irie H, Tajima T, Asayama Y, Hirakawa M, Ishigam K, Naito S, Honda $\mathrm{H}$ et al (2008) Usefulness of apparent diffusion coefficient map in diagnosing prostate carcinoma: correlation with stepwise histopathology. J Magn Reson Imaging 27:132-139

24. Tan CH, Wang J, Kundra $V$ et al (2011) Diffusion weighted imaging in prostate cancer. Eur Radiol 21(3):593-603

25. Greer MD, Brown AM, Shih JH et al (2017) Accuracy and agreement of PIRA DSv2 for prostate cancer mpMRI: a multireader study. J Magn Reson Imaging 45:579-585

26. Rosenkrantz AB, Ginocchio LA, Cornfeld D et al (2016) Interobserver reproducibility of the PI-RADS version 2 lexicon: a multicenter study of six experienced prostate radiologists. Radiology 280:793-804

27. Sasaki M, Ida M, Yamada K, Watanabe Y, Matsui M et al (2007) Standardizing display conditions of diffusion-weighted images using concurrent b0 images: a multi-vendor multi-institutional study. Magn Reson Med Sci 6 : 133-137

28. Rosenkrantz AB, Lim RP, Haghighi M, Somberg MB, Babb JS, Taneja SS et al (2013) Comparison of interreader reproducibility of the prostate imaging reporting and data system and likert scales for evaluation of multiparametric prostate MRI. AJR Am J Roentgenol 201:W612-W618

29. Rosenkrantz AB, Margolis DJ (2016) Commentary regarding the inter-reader reproducibility of PI-RADS version 2. Abdom Radiol 41:907-909

30. Vargas HA, Hotker AM, Goldman DA et al (2016) Updated prostate imaging reporting and data system (PIRADS v2) recommendations for the detection of clinically significant prostate cancer using multiparametric MRI: critical evaluation using whole-mount pathology as standard of reference. Eur Radiol 26:1606-1612

31. Kim JY, Kim SH, Kim YH, Lee HJ, Kim MJ, Choi MS et al (2014) Low-risk prostate cancer: the accuracy of multiparametric MR imagingfor detection. Radiology 271:435-444

32. Kivrak AS, Paksoy Y, Erol C, Koplay M, Ozbek S, Kara F et al (2013) Comparison of apparent diffusion coefficient values among differentMRI platforms: a multicenter phantom study. Diagn Interv Radiol 19:433-437

33. Sasaki M, Yamada K, Watanabe Y et al (2008) Variability in absoluteapparent diffusion coefficient values across different platformsmay be substantial: a multivendor, multi-institutional comparisonstudy. Radiology 249:624-630 
34. Lim HK, Kim JK, Kim KA, Cho KS et al (2009) Prostate cancer: apparent diffusion coefficient map with T2-weighted images for detection-a multireader study. Radiology 250:145-151

35. Katahira K, Takahara T, Kwee TC et al (2011) Ultrahigh- b-value diffusionweighted MR imaging for the detection of prostate cancer: evaluation in 201 cases with histopathological correlation. Eur Radiol 21:188-196

36. Haider MA, van der Kwast TH, Tanguay J et al (2007) Combined T2weighted and diffusion-weighted MRI for localization of prostate cancer. AJR Am J Roentgenol 189:323-328

37. Cobelli FD, Ravelli S, Esposito A, Giganti R, Gallina A, Montorsi R, Maschio A et al (2015) Apparent diffusion coefficient value and ratio as noninvasive potential biomarkers to predict prostate cancer grading: comparison with prostate biopsy and radical prostatectomy specimen. AJR Am J Roentgenol 204:550-557

38. Doo KW, Sung DJ, Park BJ et al (2012) Delectability of low and intermediate or high risk prostate cancer with combined T2-weighted and diffusionweighted MRI. Eur Radiol 22:1812-1819

39. Somford DM, Hambrock T, Hulsbergen-van de Kaa CA et al (2012) Initial experience with identifying high-grade prostate cancer using diffusionweighted MR imaging (DWI) in patients with a Gleason score $\leq 3+3=6$ upon schematic TRUS-guided biopsy: a radical prostatectomy correlated series. Investig Radiol 47:153-158

40. Cornud F, Delongchamps NB, Mozer P et al (2012) Value of multiparametric MRI in the work-up of prostate cancer. Curr Urol Rep 13:82-92

41. Woodfield CA, Tung GA, Grand DJ et al (2010) Diffusion-weighted MRI of peripheral zone prostate cancer: comparison of tumor apparent diffusion coefficient with Gleason score and percentage of tumor on core biopsy. Am J Roentgenol 194:316-322

42. Nowak J, Malzahn U, Baur ADJ et al (2014) The value of ADC, T2 signal intensity and a combination of both parameters to assess Gleason score and primary Gleason grades in patients with known prostate cancer. Acta Radiol 57(1):107-114

43. Caivano R, Villonio A, D' Antuono F, Gioioso M, Rabasco P, lannelli G, Zandolino A, Lotumolo A, Dinardo G, Macarini L et al (2015) Diffusion weighted imaging and apparent diffusion coefficient in 3 tesla magnetic resonance imaging of breast lesions. Cancer Investig 33:159-164. https://doi. org/10.3109/07357907.2015.1019674

44. Padhani AR, Liu G, Koh DM et al (2009) Diffusion weighted magnetic resonance imaging as a cancer biomarker: consensus and recommendations. Neoplasia 11(2):102-125

\section{Publisher's Note}

Springer Nature remains neutral with regard to jurisdictional claims in published maps and institutional affiliations.

\section{Submit your manuscript to a SpringerOpen ${ }^{\circ}$ journal and benefit from:}

- Convenient online submission

- Rigorous peer review

- Open access: articles freely available online

- High visibility within the field

- Retaining the copyright to your article

Submit your next manuscript at $\boldsymbol{\nabla}$ springeropen.com 